\title{
Robust estimation of mean and covariance for longitudinal data with dropouts
}

\author{
Guoyou Qin ${ }^{1}$, Zhongyi Zhu² \\ ${ }^{1}$ Department of Biostatistics, School of Public Health \\ and Key Laboratory of Public Health Safety, Fudan University, Shanghai, 200032, China \\ ${ }^{2}$ Department of Statistics, Fudan University, Shanghai, 200433, China
}

This file contains supplementary material for this paper. The material consists of the description of the asymptotic normality of $\hat{\beta}_{f}$, the proof of $E\left(R_{i j} \psi\left(r_{i j}\right)\right)=0$ and the proof of Theorem 1.

Lemma 1 Under conditions (C.1) - (C.3), $\sqrt{n}\left(\hat{\beta}_{f}-\beta_{0}\right)$ converges to $N\left(0, P^{-1} Q P^{-1}\right)$ in distribution.

Using regular procedure, it is not difficult to derive the result of Lemma 1 which is thus omitted here.

Lemma 2 Under the missing mechanism of MAR and the assumption that $\epsilon_{i}$ follow elliptical distribution, $E\left(R_{i j} \psi\left(r_{i j}\right)\right)=0$ where $r_{i j}=\left(Y_{i j}-D_{i j}^{T} \theta_{0}\right) / b_{j}$.

Proof: Note that if $\epsilon_{i}$ follow elliptical distribution with mean zero, then $e_{i}=C \epsilon_{i}$ also follow elliptical distribution with mean zero. Denote $\tilde{e}_{i j}=\left(e_{i 1}, \cdots, e_{i j-1}\right)^{T}$ and $\tilde{Y}_{i j}=\left(Y_{i 1}, \cdots, Y_{i j-1}\right)^{T}$. Thus we have

$$
\begin{aligned}
E\left(R_{i j} \psi\left(r_{i j}\right)\right) & =E\left\{E\left(R_{i j} \psi\left(r_{i j}\right) \mid e_{i}, Y_{i}\right)\right\} \\
& =E\left\{E\left(R_{i j} \mid e_{i}, Y_{i}\right) \psi\left(e_{i j} / b_{j}\right)\right\}
\end{aligned}
$$


Under MAR, the probability of being observed only depends on the observed values, i.e., its previously observed values in the case of dropouts. Thus, $E\left(R_{i j} \mid e_{i}, Y_{i}\right)=E\left(R_{i j} \mid \tilde{e}_{i j}, \tilde{Y}_{i j}\right)$. Denote $\pi_{i j}\left(\tilde{e}_{i j}, \tilde{Y}_{i j}\right)=E\left(R_{i j} \mid \tilde{e}_{i j}, \tilde{Y}_{i j}\right)$. Then we have

$$
\begin{aligned}
E\left[E\left\{\left(R_{i j} \mid e_{i}, Y_{i}\right) \psi\left(e_{i j} / b_{j}\right)\right\}\right] & =E\left[\pi_{i j}\left(\tilde{e}_{i j}, \tilde{Y}_{i j}\right) \psi\left(e_{i j} / b_{j}\right)\right] \\
& =E\left[E\left\{\pi_{i j}\left(\tilde{e}_{i j}, \tilde{Y}_{i j}\right) \psi\left(e_{i j} / b_{j}\right) \mid \tilde{e}_{i j}, \tilde{Y}_{i j}\right\}\right] \\
& =E\left[\pi_{i j}\left(\tilde{e}_{i j}, \tilde{Y}_{i j}\right) E\left\{\psi\left(e_{i j} / b_{j}\right) \mid \tilde{e}_{i j}, \tilde{Y}_{i j}\right\}\right] .
\end{aligned}
$$

Since $e_{i}$ follow elliptical distribution with mean zero and its covariance are identity matrix, according to the property of elliptical distribution, we have $E\left(e_{i j} \mid \tilde{e}_{i j}\right)=0$. Thus, $E\left(\psi\left(e_{i j} / b_{j}\right) \mid \tilde{e}_{i j}, \tilde{Y}_{i j}\right)=0$ considering that elliptical distribution is symmetric. Therefore, $E\left(R_{i j} \psi\left(r_{i j}\right)\right)=0$. We refer to Gómez, E.(2003) for details on the property of elliptical distribution.

\section{Proof of Theorem 1}

Let us recall the robust estimating equation

$$
\sum_{i=1}^{n} \hat{D}_{i}^{T} V_{i}^{-1 / 2} W_{i} R_{i} \psi\left(V_{i}^{-1 / 2}\left(Y_{i}-\hat{D}_{i} \theta\right)\right)=0
$$

Using the Taylor expansion, we can get

$$
n^{1 / 2}\left(\hat{\theta}-\theta_{0}\right)=\left\{\frac{1}{n} \sum_{i=1}^{n} \hat{D}_{i}^{T} V_{i}^{-1 / 2} W_{i} R_{i} \dot{\psi}\left(\hat{r}_{i}\right) \hat{D}_{i}\right\}^{-1}\left\{n^{-1 / 2} \sum_{i=1}^{n} \hat{D}_{i}^{T} V_{i}^{-1 / 2} W_{i} R_{i} \psi\left(\hat{r}_{i}\right)\right\}+o_{p}(1),
$$

where $\hat{r}_{i}=V_{i}^{-1 / 2}\left(Y_{i}-\hat{D}_{i} \theta_{0}\right)$.

By Lemma 1, $\left\|\hat{\beta}_{f}-\beta_{0}\right\|=O_{p}\left(n^{-1 / 2}\right)$, so $\left\|\hat{D}_{i}-D_{i}\right\|=O_{p}\left(n^{-1 / 2}\right)$. Then it is not difficult to show that

$$
\left\|\frac{1}{n} \sum_{i=1}^{n} \hat{D}_{i}^{T} V_{i}^{-1 / 2} W_{i} R_{i} \dot{\psi}\left(\hat{r}_{i}\right) \hat{D}_{i}\right\|=\left\|\frac{1}{n} \sum_{i=1}^{n} D_{i}^{T} V_{i}^{-1 / 2} W_{i} R_{i} \dot{\psi}\left(r_{i}\right) D_{i}\right\|+o_{p}(1) .
$$

Next, we shall show the asymptotic normality of the term $n^{-1 / 2} \hat{D}_{i}^{T} V_{i}^{-1 / 2} W_{i} R_{i} \psi\left(\hat{r}_{i}\right)$.

$$
\begin{aligned}
& n^{-1 / 2} \sum_{i=1}^{n} \hat{D}_{i}^{T} V_{i}^{-1 / 2} W_{i} R_{i} \psi\left(\hat{r}_{i}\right) \\
= & n^{-1 / 2} \sum_{i=1}^{n} \sum_{j=1}^{m} \hat{D}_{i j}\left(w_{i j} R_{i j} / b_{j}\right) \psi\left(\hat{r}_{i j}\right) \\
= & n^{-1 / 2} \sum_{i=1}^{n} \sum_{j=1}^{m}\left\{\left(\hat{D}_{i j}-D_{i j}\right)\left(w_{i j} R_{i j} / b_{j}\right) \psi\left(\hat{r}_{i j}\right)+D_{i j}\left(w_{i j} R_{i j} / b_{j}\right) \psi\left(\hat{r}_{i j}\right)\right\}
\end{aligned}
$$




$$
\begin{aligned}
= & n^{-1 / 2} \sum_{i=1}^{n} \sum_{j=1}^{m}\left(\hat{D}_{i j}-D_{i j}\right)\left(w_{i j} R_{i j} / b_{j}\right) \psi\left(r_{i j}\right) \\
& +n^{-1 / 2} \sum_{i=1}^{n} \sum_{j=1}^{m}\left(\hat{D}_{i j}-D_{i j}\right)\left(w_{i j} R_{i j} / b_{j}\right) \dot{\psi}\left(r_{i j}^{*}\right)\left(\hat{r}_{i j}-r_{i j}\right) \\
& +n^{-1 / 2} \sum_{i=1}^{n} \sum_{j=1}^{m} D_{i j}\left(w_{i j} R_{i j} / b_{j}\right) \psi\left(r_{i j}\right) \\
& +n^{-1 / 2} \sum_{i=1}^{n} \sum_{j=1}^{m} D_{i j}\left(w_{i j} R_{i j} / b_{j}\right) \dot{\psi}\left(r_{i j}^{*}\right)\left(\hat{r}_{i j}-r_{i j}\right) \\
\doteq & A_{1}+A_{2}+A_{3}+A_{4},
\end{aligned}
$$

where $r_{i j}^{*}=\left(Y_{i j}-D_{i j}^{*} \theta_{0}\right) / b_{j}$ and $D_{i j}^{*}$ are evaluated at $\beta^{*}$ which is between the line $\beta_{0}$ and $\hat{\beta}_{f}$.

Since $\left\|\hat{\beta}_{f}-\beta_{0}\right\|=O_{p}\left(n^{-1 / 2}\right)$ and thus $\hat{\epsilon}_{i j}-\epsilon_{i j}=O_{p}\left(n^{-1 / 2}\right)$, it is easy to verify that $\left\|A_{1}\right\|=o_{p}(1)$ and $\left\|A_{2}\right\|=o_{p}(1)$.

We first consider $A_{4}$.

$$
\begin{aligned}
A_{4} & =n^{-1 / 2} \sum_{i=1}^{n} \sum_{j=1}^{m} D_{i j}\left(w_{i j} R_{i j} / b_{j}\right) \dot{\psi}\left(r_{i j}^{*}\right)\left(\hat{r}_{i j}-r_{i j}\right) \\
& =n^{-1 / 2} \sum_{i=1}^{n} \sum_{j=1}^{m} D_{i j}\left(w_{i j} R_{i j} / b_{j}\right) \dot{\psi}\left(r_{i j}^{*}\right)\left(D_{i j}-\hat{D}_{i j}\right) \theta_{0} \\
& =n^{-1 / 2} \sum_{i=1}^{n} \sum_{j=1}^{m} D_{i j}\left(w_{i j} R_{i j} / b_{j}\right) \dot{\psi}\left(r_{i j}\right)\left(D_{i j}-\hat{D}_{i j}\right) \theta_{0}+o_{p}(1)
\end{aligned}
$$

Since $D_{i j}=\left(X_{i j}^{T}, F_{i j}^{T}\right)^{T}$, we have $\left(D_{i j}-\hat{D}_{i j}\right) \theta_{0}=-\sum_{k=1}^{j-1} \varsigma_{j k} X_{i k}^{T}\left(\hat{\beta}_{f}-\beta_{0}\right)$. Moreover, using regular procedure, it is not difficult to show

$$
\hat{\beta}_{f}-\beta_{0}=\left\{\sum_{i=1}^{n} X_{i 1} w_{i 1} \dot{\psi}\left(r_{i 1}\right) X_{i 1}^{T}\right\}^{-1} \sum_{i=1}^{n} X_{i 1} w_{i 1} \psi\left(r_{i 1}\right)+o_{p}\left(n^{-1 / 2}\right) .
$$

Then we have

$$
\begin{aligned}
A_{4}= & -n^{-1 / 2} \sum_{i=1}^{n} \sum_{j=1}^{m} D_{i j}\left(w_{i j} R_{i j} / b_{j}\right) \dot{\psi}\left(r_{i j}^{*}\right) \sum_{k=1}^{j-1} \varsigma_{j k} X_{i k}^{T}\left(\hat{\beta}_{f}-\beta_{0}\right) \\
= & -n^{-1 / 2} \sum_{i=1}^{n} \sum_{j=1}^{m} D_{i j}\left(w_{i j} R_{i j} / b_{j}\right) \dot{\psi}\left(r_{i j}\right) \sum_{k=1}^{j-1} \varsigma_{j k} X_{i k}^{T}\left\{\sum_{i=1}^{n} X_{i 1} w_{i 1} \dot{\psi}\left(r_{i 1}\right) X_{i 1}^{T}\right\}^{-1} \sum_{i=1}^{n} X_{i 1} w_{i 1} \psi\left(r_{i 1}\right) \\
& +o_{p}(1) \\
= & -n^{-1 / 2} \sum_{i=1}^{n}\left[\left\{\sum_{i=1}^{n} \sum_{j=1}^{m} D_{i j}\left(w_{i j} R_{i j} / b_{j}\right) \dot{\psi}\left(r_{i j}\right) \sum_{k=1}^{j-1} \varsigma_{j k} X_{i k}^{T}\right\}\left\{\sum_{i=1}^{n} X_{i 1} w_{i 1} \dot{\psi}\left(r_{i 1}\right) X_{i 1}^{T}\right\}^{-1} X_{i 1} w_{i 1} \psi\left(r_{i 1}\right)\right]
\end{aligned}
$$




$$
+o_{p}(1)
$$

and

$$
\begin{aligned}
A_{3}+A_{4}= & n^{-1 / 2} \sum_{i=1}^{n}\left[\left\{\sum_{j=1}^{m} D_{i j}\left(w_{i j} R_{i j} / b_{j}\right) \psi\left(r_{i j}\right)\right\}\right. \\
& \left.-\left\{\sum_{i=1}^{n} \sum_{j=1}^{m} D_{i j}\left(w_{i j} R_{i j} / b_{j}\right) \dot{\psi}\left(r_{i j}\right) \sum_{k=1}^{j-1} \varsigma_{j k} X_{i k}^{T}\right\}\left\{\sum_{i=1}^{n} X_{i 1} w_{i 1} \dot{\psi}\left(r_{i 1}\right) X_{i 1}^{T}\right\}^{-1} X_{i 1} w_{i 1} \psi\left(r_{i 1}\right)\right]+o_{p}(1) .
\end{aligned}
$$

Then according to Lemma 2 and condition (C.5), and by central limit theorem, we obtain

$$
A_{3}+A_{4} \rightarrow N(0, K),
$$

and in the end,

$$
n^{1 / 2}\left(\hat{\theta}-\theta_{0}\right) \rightarrow N(0, \Omega),
$$

where $\Omega=S^{-1} K S^{-1}$. 